\title{
Modelos de gestión en instituciones hospitalarias*
}

\section{Management Models for Hospitals}

\section{Modelos de gestão em instituições hospitalares}

Fecha de recepción: 30 de mayo de 2018. Fecha de aprobación: 31 de octubre de 2018.

Fecha de publicación: 27 de mayo de 2019

\author{
DOI: https://doi.org/10.11144/Javeriana.rgps18-36.mgih \\ Luis Enrique Perea Vásqueza \\ Universidad Simón Bolívar, Colombia \\ ORCID: 0000-0002-2724-676X \\ Indiana Luz Rojas Torres \\ Universidad Simón Bolívar, Colombia \\ ORCID: 0000-0002-2601-2363
}

Cómo citar este artículo: Perea Vásquez LE, Rojas Torres IL. Modelos de gestión en instituciones hospitalarias. Revista Gerencia y Políticas de Salud. 2019;18(36). https://doi.org/10.11144/Javeriana.rgps18-36.mgih

Artículo de investigación

Autor de correspondencia. Correo electrónico: lupevas@ hotmail.com 


\section{Resumen}

El presente trabajo explora los modelos de gestión en instituciones hospitalarias. Es una investigación exploratoria con un diseño de investigación documental, dado que se realizó la revisión de los distintos modelos a través de la historia, haciendo una revisión exhaustiva del modelo de la nueva gestión pública del Centro Latinoamericano de Desarrollo (CLAD). Se concluye que en las instituciones hospitalarias pertenecientes al Estado, este modelo se puede llevar a cabo con éxito, si hay una profesionalización de la alta burocracia como un punto estatal estratégico y, además, si se cuenta con el apoyo y la movilización en el proceso de reforma gerencial del Estado, lo cual permitirá transformar la actual estructura de la administración pública.

Palabras clave: política pública, gestión, hospital, gobierno, salud, políticas de salud

\section{Abstract}

This work explores the management models for hospitals. It is an exploratory research with a documentary design applied in the review of the different management models throughout the history and including an in-depth inquiry on the new public management model by the Latin-American Development Center (CLAD). It is concluded that in the State-owned hospitals this model can be successfully implemented whenever the individuals in the top management become professionals. This effort should be taken as a strategic measure by the State in addition to the support and mobilization towards a managerial reform process. All this will allow transforming the current structure of the public management.

Keywords: public policy, management, hospital, government, health, health policies

\section{Resumo}

O presente trabalho explora modelos de gestado em instituições hospitalares. Trata-se de uma pesquisa exploratória com delineamento de pesquisa documental, dado que realizou revisão dos diferentes modelos ao longo da história, fazendo revisão exaustiva do modelo da nova gestão pública do Centro Latino-americano de Desenvolvimento (CLAD). Conclui-se que nas instituições hospitalares pertencentes ao Estado, esse modelo pode ser realizado com sucesso, se houver uma profissionalização da alta burocracia como ponto estadual estratégico e, além disso, se houver apoio e mobilização no processo de reforma gerencial do Estado, o qual permitirá transformar a atual estrutura da administração pública.

Palavras chave: política pública, gestão, hospital, governo, saúde, políticas de saúde 


\section{Introducción}

En este mundo lleno de cambios e incertidumbre, lo que ahora se comenta ya es historia. La globalización de la economía, la apertura de los mercados, el desarrollo de la tecnología de la información y de las telecomunicaciones están destruyendo las barreras tradicionales. Los negocios han eliminado las barreras geográficas y territoriales del pasado y, como consecuencia de todo lo anterior, se producirá una nueva definición y visión de los mercados. Las organizaciones tendrán que volcarse hacia los clientes y la calidad en los productos y servicios, lo que se constituirá en la verdadera ventaja competitiva.

Para facilitar todos estos procesos, el Estado se modernizará, se privatizarán las actividades no sensibles a la seguridad nacional y los particulares tendrán que prepararse para asumir la gestión de servicios y tareas antes en mano de la nación. En este marco, la competencia será más agresiva, el cambio y la innovación serán las constante y la capacidad de respuesta será un elemento estratégico fundamental. Aparecerá entonces la reingeniería organizacional como estrategia para dinamizar las organizaciones, hacerlas más flexibles, más planas, más orientadas al mercado y al cliente.

Las instituciones hospitalarias públicas, en el marco de la nueva gestión pública, deberán aplicar aquellas reformas que garanticen una mayor efectividad y eficiencia. Estas empresas operan en mercados imperfectos, deberán tener un manejo flexible, descentralización operativa y autonomía relativa, combinados en dosis prudentes con la planificación y los controles, junto con más y mejor inversión constituye el marco estratégicos para estas empresa (1).

El objetivo central de este artículo es presentar un análisis histórico de los modelos de gestión, gobernanza y financiación de los hospitales públicos colombianos para contrastarlos con los modelos desarrollados en otros países, que permitan orientar una propuesta de cambio en este tema, concretamente en Colombia.

\section{Metodología}

Esta revisión se realizó a través de la búsqueda en las bases de datos de Redalyc, Scielo, Elsevier, Pubmed, Ovid y Medline, utilizando las palabras clave: política pública, gestión, hospital, gobierno, salud y políticas de salud. Como criterios de inclusión se consideraron los elementos de los sistemas y los modelos de salud que se han dado a través de la historia, haciendo una revisión exhaustiva del modelo de la nueva gestión pública del Centro 
Latino Americano de Desarrollo (CLAD), no como elemento aislado, sino como parte de una revisión teórica que permite destacar los rasgos que asumen procesos, tales como: toma de decisión, administración de personal, prestación y financiamiento de servicios. Se excluyeron revisiones que no hacían referencia a los modelos de salud. Posteriormente, se contrastan los modelos de gestión colombianos, a fin de construir una propuesta en otros entornos fuera de Colombia. Finalmente, se reflexiona sobre la necesidad de la nueva gestión pública.

\section{Consideraciones teóricas sobre gestión de las organizaciones}

En este apartado se discuten los componentes de gestión desde las organizaciones (burocrático, burocrático-populista, tecnocrático y nueva gestión pública).

La burocracia se define por ser una estructura administrativa y de personal de una organización. Las entidades empresariales, religiosas, docentes y oficiales exigen unos abundantes recursos humanos, ordenados según esquemas jerárquicos para desempeñar sus tareas especializadas basadas en el reglamento interno. El término se utiliza principalmente para referirse a la administración pública, pero hoy se utiliza de manera peyorativa para referirse a la pérdida de tiempo e ineficacia (2).

Más que una definición de la burocracia como órgano institucional o parte integral de lo que es la función pública, es más bien una descripción de la estructura burocrática, de cómo se compone. Niskanen ofrece una definición de la burocracia: las burocracias son organizaciones no lucrativas, es decir, sin afán de lucro; al menos una parte de la burocracia está financiada por una asignación periódica o por una subvención (3).

Weber con referencia al tipo de organización burocrática propone recurrir a los famosos ideales tipo, los cuales son capaces de responder a la dificultad particular de las ciencias humanas que explican tanto los fenómenos generales como las singularidades espaciales y temporales (4). Un ideal tipo, según lo concebido por el autor, es una contribución a la epistemología de las ciencias sociales por el método de comprensión-comparación que este permite. Para Weber los ideales tipo, la dominación legal y burocrática sirven para formar conceptos, cuyo rol y uso llevaran por comparación al estudio y a la comprensión de situaciones y géneros históricamente individualizados. 
El tipo más puro de dominación legal es aquel que se ejerce por medio de un cuadro administrativo burocrático, pero sus facultades de mando son también "competencias legales". Esto puede aplicarse igualmente a establecimientos económicos, caritativos o cualquiera otro de carácter privado que persiga fines materiales o ideales, y a asociaciones políticas o hierocracias. Así, por ejemplo, las burocracias en las clínicas privadas son en principio de igual carácter que las de los hospitales de fundaciones u órdenes religiosas.

Más allá de la situación de poder condicionada por el saber de la especialidad, la burocracia tiene la tendencia a acrecentar aún más el poder por medio del saber de servicio, siendo el empresario capitalista la única instancia inmune a lo ineludible de la dominación científico-racional de la burocracia. Todos los demás, en las asociaciones de masas, están irremisiblemente sometidos al imperio burocrático, de igual forma que la producción en masas lo está al dominio de las máquinas de precisión (5).

El poder de la burocracia se ha comparado usualmente con el que ejercen otros actores políticos y económicos, ya sean partidos políticos, el parlamento, la presidencia, los sindicatos, los grupos corporativos empresariales y otros. La literatura ha prestado gran atención a este tema desde que en los años setenta y ochenta, los marxistas redescubrieron la noción de "autonomía relativa del Estado" de Marx en el 18 Brumario de Luis Bonaparte, y desde que los neoweberianos comenzaron a hacer una interpretación más política al tipo ideal de burocracia desarrollado por Weber (6).

En cuanto a las agencias burocráticas, estas son organizaciones no lucrativas financiadas al menos parcialmente por una entidad pública, en general, el Gobierno. Es decir, las agencias burocráticas ofrecen un servicio o producto financiado por un presupuesto público; normalmente estos servicios son muy difíciles de cuantificar e incluso de definir. Ello ocasiona que el financiador no tenga una información completa sobre el presupuesto necesario para la realización de la actividad. En estas condiciones, la teoría macroeconómica de la burocracia predice inferencia económica. En términos hospitalarios, la gestión del hospital burocrático queda en manos de los gerentes o los médicos, quienes pueden tener objetivos alejados de la eficiencia productiva (7).

El sometimiento de las instituciones de salud a la influencia de los partidos políticos se identifica como uno de los fenómenos que más afectan el desarrollo de estas instituciones y el logro de los objetivos del sistema de salud, 
lo cual se agrava por un proceso de descentralización sin un direccionamiento y asistencia técnica efectiva, de lo cual los aseguradores privados y el clientelismo ha sacado provecho. Este aspecto está ligado al nexo histórico entre Estado e instituciones públicas, partidos políticos y élite económica. Por lo tanto, la carencia de sentido de lo público impide el desarrollo de los servicios de salud enfocado en la justicia social (8). A este respecto, María Teresa Uribe sostiene:

[...] en América Latina, de la que Colombia no es la excepción, se percibe un amplio malestar con los parlamentos y congresos, considerados por la opinión pública como fuentes de corrupción, ineficacia y desgreño [...], herencia de una arcaica democracia formal, [...] que afecta la moralidad del Estado y la Nación [...] En los partidos políticos se destaca su creciente incapacidad para encauzar la multiplicidad y diversidad de las demandas sociales, la pérdida de identidad política de los ciudadanos con ellos, así mismo su carácter de maquinarias electorales, orientadas hacia el uso privado y particular de las clientelas que las constituyen. (9)

Los hospitales configuran aquel tipo de organizaciones que Henry Mintzberg llamó burocracias profesionales, donde el poder es descentralizado internamente y cada jefe de servicio actúa como autoridad indiscutida. Sin embargo, al existir dos tipos de tareas diferenciadas, las organizaciones hospitalarias avanzadas configuran la yuxtaposición de dos organizaciones: una burocracia tradicional (o mecánica) y una burocracia profesional. Para los estructuralistas, aumentar la racionalidad del hospital en la toma de decisiones implica maximizar el carácter del hospital como burocracia mecánica. Esto se logra verticalizando lo más posible la institución, implementando normas y procedimientos allí donde sea posible y regularizando y estandarizando todos los procesos (10). En ese sentido, resulta fundamental descentralizar el poder hacia el interior del hospital, involucrando progresivamente a los médicos en las decisiones administrativas.

Al analizar la organización interna del hospital, esta reproduce el paradigma burocrático weberiano predominante, ya que en la cúspide de la organización se ubicaba el director médico, mientras que la base de la pirámide hospitalaria la ocupaban los operadores de servicios clínicos que se brindarían a los pacientes. Fueron ejemplo de esta burocracia pública los sistemas de salud en América Latina y gran parte del mundo y con ellos inmersos las 
instituciones hospitalarias, las cuales se caracterizaron por una fuerte integración de la financiación y producción o provisión de servicios de salud.

Estas organizaciones sanitarias, así configuradas, están regidas por el principio de jerarquía, sometidas al derecho público en todos sus ámbitos y sujetas a un fuerte proceso de centralización en la toma de decisiones. Su actuación en régimen de cuasimonopolio, así como la obtención de sus ingresos mediante transferencias de los presupuestos públicos, y no mediante la venta de sus servicios, disminuye los incentivos para una conducta eficiente tanto de directivos como de profesionales y trabajadores (11). De forma coherente con este modelo burocrático, los servicios de salud están sometidos al derecho administrativo en lo que concierne a su ámbito de la gestión, que se orienta fundamentalmente al cumplimiento de la legalidad y no a la eficiencia en el uso de los recursos públicos.

En el modelo burocrático-populista existe una convergencia a la planificación normativa y estratégica. En la estructura organizativa se destaca una alta división del trabajo, con grandes estructuras organizativas, numerosos niveles jerárquicos y departamentos con tareas y funciones organizadas y especializadas. Es un modelo administrativo público iniciado en la democracia, puesto que impone una colaboración del aparato público a través del gasto público para el logro de sus objetivos (12).

En América Latina, el modelo burocrático-populista es un fenómeno asociado al Estado de bienestar keynesiano, cuyo objetivo fundamental es la intervención en la economía para lograr el beneficio de la colectividad mediante la búsqueda del pleno empleo, fomento de los servicios de bienestar social e integración cultural y social. Por su parte, en Venezuela se manifiesta a partir del proceso democratizador experimentado en el país a finales de la década de los cincuenta, el cual pretendía promover el crecimiento acelerado de la economía, el impulso al sector industrial, las inversiones en infraestructura de carácter social, entre otros aspectos (13).

La instauración de este modelo se dio por el avance de la economía de sustitución de las importaciones y el crecimiento de los partidos políticos, particularmente de partidos de corte populista caracterizados por una marcada inclinación hacia la personalización del liderazgo que favorece una innegable orientación autoritaria, en detrimento de un régimen orientado por valores éticos que permiten un desarrollo diferente del aparato público. 
Se produjo un estilo de hacer política que privilegiaba el ascenso de los puestos de la dirección estatal por la vía de la influencia partidista, como la captura de todo el aparato público por parte de los partidos políticos. Los principales sujetos de decisión son los militantes de los partidos políticos y empresarios que se ubican en los niveles más altos del aparato público, logrando de esta manera dominar los procesos de toma de decisiones e incluso la selección del personal directivo, tales como ministros, presidentes de empresas públicas y gerentes de distintos niveles (14).

Las políticas en salud diseñadas e implementadas por el Estado populista forman parte de las políticas sociales, las cuales se caracterizaron durante el proceso de consolidación de la democracia representativa, por ser de corte universalista, es decir, no privilegiaron a sectores específicos, sino que estuvieron dirigidas en lo formal a satisfacer la demanda de todos los sectores y grupos sociales, con el fin de legitimar las acciones democráticas y combatir de esta manera lo que consideraban la "amenaza" del comunismo. Desde lo real, las políticas universalistas en salud favorecieron a sectores vinculados al puntofijismo y a las burocracias estatales (15).

El financiamiento del servicio descansa en el aparato del Estado, el cual destina recursos de manera desmesurada y genera altísimas erogaciones que no corresponden con los servicios prestados. Esta situación, aunada a los ingresos fiscales, estimuló la asociación entre grupos médicos, permitió la creación de nuevas instituciones en el área, como fueron los grandes hospitales de tipo empresarial, y esto trajo como consecuencia el desplazamiento del sector público por parte del privado, mientras en el público se afianzó la estructura sanitaria como espacio clientelar-partidista que profundizó la corrupción administrativa (16).

Como se puede observar, se produce la implantación de un modelo que privilegia la medicina de alto costo y alta tecnología y que deja atrás a la atención primaria en salud en lo concerniente a la promoción y prevención, con el agravante de disminuir aún más los recursos de salud con los que se cuenta para la atención de las personas. La mayoría de estos servicios no está en los planes de beneficios de los sistemas de salud, lo cual hace que se imponga un modelo individual-curativo de alto consumo y que el gasto en bolsillo de las personas aumente. 
Esta etapa continúa con rasgos burocrático-weberianos. Los directores o gerentes de las instituciones hospitalarias eran nombrados por recomendación política, lo cual influía en la gestión, el financiamiento, la provisión y venta de servicios de salud. La selección de personal de libre nombramiento y remoción que formaba parte de estas instituciones era por clientelismo político, e inclusive los contratistas que realizaban las obras de adecuación, mantenimiento $\mathrm{u}$ obras nuevas eran escogidos por recomendación del político de turno, lo cual caracterizó a las instituciones como ineficientes, clientelistas y de alta corrupción administrativa.

En relación con la tecnocracia, se podría designar a Francis Bacon como el primer filósofo preocupado por la aplicación de conocimiento científico en la vida diaria. Este pensador inglés actuaba en el marco de una época: “se observó un continuo incremento del poder y prestigio de la ciencia y de la innovación técnica en paralelo a una declinación del prestigio de las aristocracias y de la instituciones religiosas". Posteriormente, Thorteins Veblen promovía que ingenieros científicamente capacitados remplazaran a industriales y hombres de negocios inhabilitados para guiar correctamente el proceso productivo. Fue así cómo, con este propósito, se creó la Technical Alliance to Promote the Technocracy, institución que desapareció en 1922 y fue remplazada por el Committee of Technocracy, en cuyo texto se afirma: "la tecnocracia es la ciencia aplicada al orden social. La tecnocracia, entonces, se ocupa de determinar la realidad más probable en el campo de la ciencia social, es decir, la determinación de su estado más probable; tiene que ver, primariamente, con esa parte del mecanismo social que se encarga de la producción y distribución de bienes y servicios, pero tiene implicaciones de mayores alcances aun" (17).

La tecnocracia, que tuvo su origen en el desarrollo vertiginoso de la ciencia y la técnica en las últimas décadas, posee fundamentos conceptuales de muchas vertientes (inclusive de la administración científica), pero sobre todo del enfoque weberiano, en lo que tiene que ver con la tecnificación y preparación del funcionario. Sin embargo, la tecnocracia ha ido asumiendo una serie de características particulares, sobre todo en la racionalidad legal propia de la burocracia en la racionalidad técnica, esencia de la tecnocracia (18).

El modelo tecnocrático sigue los lineamientos del mercado y busca establecer vínculos entre el Estado y el sector privado, donde grupos de poder mediante parámetros de prestación de servicios a través de terceros o outsourcing puedan obtener un beneficio producto de una utilidad. Este 
modelo se promueve en una economía de mercado basada en la tesis del modelo neoliberal, que pasó a la reducción del Estado en el ejercicio de sus funciones, particularmente en el área social que da oportunidad al desarrollo de una política social individualista, inequitativa y con altos costos. La extrema división horizontal y vertical del trabajo, altamente compleja, rígida, inspirada en el modelo ideal weberiano, se transforma en estructura plana, con un mínimo de niveles jerárquicos. Se sustituye en el nivel directivo al hombre de partido o al recomendado político por profesionales o técnicos con orientación economicista (14).

La racionalidad económica esencial de este modelo tiene distintos significados según la jerarquía de los sujetos. Para los funcionarios administrativos de las entidades significa asumir la misión "de producir servicios de salud", de conformidad con los procesos organizacionales y el uso de los recursos públicos. Para los trabajadores de salud significa ajustar sus prácticas a parámetros administrativos, y con esto puede perder autonomía profesional. También significa el deterioro de las condiciones laborales: disminución del número de cargos con la intensificación del trabajo para los que quedan, todo esto fruto de la política de flexibilización laboral que busca la racionalización de los recursos humanos (19).

La racionalidad del mercado de los sistemas de salud no es buena, sino por el contrario, hace peligroso al sistema y las políticas de salud para el deseo de justicia social, no solo porque excluye una alta proporción de pobres, sino que también genera inequidad en el plan de beneficios de salud (20). Los procesos de selección y ascenso son realizados tomando en cuenta los méritos; es lo que se ha denominado meritocracia, esta se constituye en un mecanismo de legitimación de la tecnocracia. Bajo esquemas tecnocráticos, la participación directa del Estado en la producción de bienes y servicios se reduce, se pone en práctica la privatización en áreas importantes de la administración pública, como la salud. La privatización se da con el argumento de contribuir a elevar los niveles de eficiencia de las empresas públicas y los servicios sociales y mejorar la calidad de la atención (14).

Con la privatización se introducen elementos del mercado como el cobro por servicio, lo que implica la transferencia de la responsabilidad del financiamiento del sector público al usuario para que este pague directamente. Y esto va en detrimento de principios como la gratuidad y la equidad, puesto 
que un gran número de usuarios por carecer de recursos ven quebrantado el disfrute de los servicios (21).

La adopción de la descentralización que tiene lugar en este periodo puede ser asimilada como expresión del modelo tecnocrático, en vista de que busca la reducción del aparato del Estado, la eficiencia del gasto y el incremento de la calidad y la productividad de los servicios mediante su traslado a la sociedad civil. Así mismo, permite la incorporación de otros actores, incluyendo la comunidad en el proceso de toma de decisiones, mostrando de esta manera un tímido proceso democratizador (16).

Además, la privatización "está asociada principalmente a unas disposiciones según las cuales el gobierno delega la prestación de los servicios y el cobro de los fondos para financiarlos, esto es el bien o servicio pasa a regirse por las leyes del mercado, excluyendo o permitiendo su consumo en función de criterios de razonabilidad económica" (22).

En este marco tecnocrático, marcado por la privatización, se acaba el monopolio del Estado en la prestación de los servicios de salud y se le dan herramientas al sector privado para que compita en la provisión y venta de servicios de salud. Las instituciones hospitalarias se convierten en entidades con autonomía propia y administrativa y presupuestaria, regidas por el derecho privado. Su financiamiento ya no va a depender de las transferencias ordinarias, sino de la venta de servicios de salud. El Estado pasa a ser regulador del sistema y a ejercer la inspección, vigilancia y control del sistema de salud.

Estos modelos gerencialitas desarrollados por las instituciones hospitalarias a través de contrato programa o contrato de gestión, la gestión clínica y la incorporación de la filosofía de gestión empresarial como gestión por proceso o gestión por competencia, generan un marco coherente para producir mecanismos de incentivos y alejarse progresivamente de los modelos burocráticos de producción de servicios sanitarios (11). Precisamente, la gestión de procesos busca reducir la variabilidad que aparece habitualmente cuando se producen o prestan determinados servicios y trata de eliminar las ineficiencias asociadas a la repetitividad de las acciones o actividades y al consumo inapropiado de recursos (23).

La reforma del Estado se convirtió en el tema central de la agenda política mundial, impulsando como modelo de desarrollo la nueva gerencia pública. 
De acuerdo con este se orientaron los procesos de reforma, cuando el modelo de Estado conformado por los países desarrollados en la posguerra, y el cual le dio vida al capitalismo como modelo propulsor de prosperidad, entró en crisis hacia fines de la década de los setenta. En un primer momento, la respuesta a la crisis fue la neoliberal-conservadora, y posteriormente para restablecer el equilibrio fiscal y equilibrar la balanza de pagos de los países en crisis, se orientó esta reforma hacia la reducción del tamaño del Estado y el predominio total del mercado (24).

Para algunos, el origen de la nueva gerencia pública se encuentra en la economía y, particularmente, en el pensamiento económico neoclásico engendrado en la escuela austriaca, así como de la opción pública (publica choice) estadounidense, donde se exalta lo privado, el individualismo y la rentabilidad. Por ello, los objetivos centrales de este modelo y sus resultados deben ser orientados hacia la privatización del Estado (25).

El término nueva gerencia pública o nueva gestión pública sirve para designar un nuevo modelo de administración pública. En palabras de Barzelay, "la nueva gerencia pública se origina como un dispositivo conceptual inventado con el propósito de estructurar la discusión académica sobre los cambios contemporáneos en la organización y el gerenciamiento de la rama ejecutiva del gobierno" (26).

La nueva gerencia pública asume distintos significados en diferentes contextos administrativos. Sin embargo, los elementos del término no son tan nuevos, se relacionan con una visión más económica y gerencial de la administración gubernamental (27). La nueva gerencia pública se enmarca en el modelo individualista de gestión que privilegia una perspectiva de mercado fundamentada en la maximización de la utilidad. Esta visión considera al Estado una estructura que provee servicios y, en tal sentido, ve al ciudadano como un cliente y busca generar un escenario de competencia e innovación para alcanzar su mejor provisión (28).

Hay dos concepciones de la nueva gerencia pública, una que discute nuevos principios administrativos tales como la contestabilidad, la elección del usuario, la transparencia y una estrecha focalización en las estructuras de incentivos. La segunda concepción se desprende de la aplicación de los principios gerenciales del sector empresarial privado al sector público, lo que implica un énfasis en la gerencia de contratos, la introducción de mecanismos de mercado ${ }^{1}$ en el sector público y la vinculación del pago con el desempeño (27). 
Más allá de su objeto, lo que verdaderamente caracteriza a la nueva gerencia pública es su aproximación al sector público, de una forma diferente a lo tradicional. Se ha buscado una mayor eficacia y eficiencia del aparato administrativo estatal trasladando al sector público. En esta misma línea de razonamiento, han argumentado que la nueva gestión pública supondría de hecho "la incorporación del estado a la era del management", y esto supone la aplicación estatal del marco institucional que ha operado en la gran empresa (29).

Así pues, la nueva gerencia pública es una visión privada de lo público, se sitúa en el mercado para sustituir el esquema burocrático, "es la implantación de la imagen empresarial de los negocios privados dentro del gobierno, cuya idea es que el sector público esté en manos de gerentes profesionales, dotados con un control activo, visible y discrecional sobre las organizaciones". Se concibe como opuesta a la administración pública y se le entiende como una transdisciplina económica que puede sustituirla benéficamente. Se trata de un modelo de dentro hacia fuera, no de fuera hacia dentro (25).

La planificación estratégica es vista como un enfoque objetivo y sistemático para la toma de decisiones en la administración pública. Como proceso gerencial, busca desarrollar y mantener una dirección estratégica que pueda alinear las metas y recursos de la organización con sus oportunidades cambiantes de mercadeo (30). En realidad, este es un proceso muy complejo que requiere un enfoque sistemático para identificar y analizar factores externos a la organización y confrontarlos con las capacidades de la administración. Es necesario señalar que los mecanismos de control impuestos por las tendencias tecnocráticas descansan sobre el control de gestión y la auditoría externa (no son categorías objeto de estudio de esta investigación), cuya base es la planificación estratégica desde el punto de vista formal, la cual tiene lugar en los niveles más altos de la organización, que son controlados por el proceso de trabajo, y por los resultados esperados (31). El estudio de la dirección estratégica se ha centrado extensamente en el sector privado más que en organizaciones públicas y sin ánimo de lucro. Bajo la corriente de la nueva gestión pública, los hospitales requieren equipos directivos con diversas características y conocimientos, deben responsabilizarse no solo del control del costo, sino también de la coordinación y el desarrollo de nuevos servicios (32). 
Es importante aclarar que en las organizaciones públicas en general, y en los hospitales en particular, la implementación de políticas y planes estratégicos no es una elección de los gerentes o directores, sino más bien estos deben implantar las políticas estratégicas, centradas en la reducción de costos y mejora de la flexibilidad organizativa, que las distintas entidades gubernamentales formulan e imponen a los hospitales a través de los planes estratégicos. De conformidad con la corriente de la nueva gestión pública, los hospitales requieren equipos directivos de diversas características y conocimientos, no solo deben responsabilizarse del control de costos, sino también de la coordinación y el desarrollo de nuevos servicios, lo cual ha traído la atención de los investigadores en dirección estratégicas (33).

El modelo gerencial se inspira en los cambios organizacionales del sector privado, se adoptan las herramientas provenientes de este por parte del sector público, se aboga por la flexibilización organizacional y en ese sentido se sustituye la forma estructural burocrático-piramidal y de normas centralizadas, con el aumento de la responsabilidad y autonomía de decisión de los gerentes, y estructuras aplanadas. Kliskberg señala (34):

En contextos de cambio permanente como el presente, una recomendación elemental es maximizar la flexibilidad de la organización a fin de dotarla de las mejores condiciones de adaptabilidad. Si se pone el énfasis en estructuras fijas, permanentes, rígidas, se está yendo en el sentido opuesto. La capacidad de reaccionar ante situaciones cambiantes va a disminuir seriamente.

Se promueve la descentralización y desconcentración organizacional, delegando a estos organismos la ejecución de funciones. Se promueve la separación entre las estructuras responsables de la formulación de políticas y los organismos descentralizados, las cuales se encargarán de su ejecución, manteniéndose control sobre los resultados obtenidos. También se señala que entre los cambios que deben producirse en la modernización está la flexibilización del trabajo, pero destacándola en dos vertientes, entre ellas la relativa a la organización del trabajo, la cual alcanza la adaptación de la estructura organizacional: “[...] cambio de una estructura piramidal a una estructura horizontal o aplanada, reduciendo significativamente los niveles jerárquicos" (35).

A este respecto: 
[...] la flexibilidad funcional, organización interna de la empresa, no fragmentación, no división de la mano de obra, movilidad interna; flexibilidad en la gestión, adopción de técnicas que permiten el uso más eficiente de los recursos materiales, financieros tecnológicos y de información para alcanzar los objetivos organizacionales de forma competitiva. (36)

En la nueva gerencia pública se intenta conformar estructuras organizativas más aplanadas, con el objeto de acercar el poder de decisión a los usuarios o clientes (empowerment), considerados actores esenciales del proceso, facilitando así una atención individualizada. Por otro lado, se facilita la circulación de la información, tanto ascendente como descendente, y el análisis de las demandas sociales y la capacidad de reacción estratégica de la organización.

Las sociedades exigen cambios organizacionales que les permitan participar en la profundización de la democracia y de esta manera trascender. En este sentido, demandan modelos organizacionales que realmente faciliten y mejoren los mecanismos de transparencia en lo que hace a la información de los actos públicos, además de ingresar al sistema de control social (29).

La reforma gerencial, derivada de la nueva gerencia pública, es un elemento clave en la profesionalización de la burocracia, que ejerce funciones en el núcleo de actividades centrales del Estado, lo que la asemeja en este aspecto al modelo weberiano. Asimismo, tener funcionarios públicos calificados y permanentemente entrenados, protegidos de las interferencias políticas, bien remunerados y motivados. La reforma de la administración pública debe ser ejecutada en tres dimensiones: (1) una dimensión institucional-legal, a través de la cual se modifican las leyes y se crean o se modifican las instituciones; (2) una dimensión cultural, basada en los cambios de los valores burocráticos a los gerenciales; y (3) una dimensión-gestión, se trata aquí de poner en práctica las nuevas ideas gerenciales y de ofrecer a la sociedad un servicio público efectivamente más barato, mejor controlado y de mejor calidad (37).

El CLAD señala que la profesionalización de la alta burocracia es el punto de partida de la reforma gerencial, y en tal sentido propone constituir un núcleo estatal estratégico, dirigido a la formulación, supervisión y regulación de las políticas, formado por una élite burocrática técnicamente preparada y motivada, y la cual deberá enfrentar procesos de negociación y de responsabilización ante el sistema político (26). 
En el actual contexto de transformaciones del Estado y de la gestión pública, sobresalen marcadas tendencias que determinan cambios sustantivos en la conceptualización, valoración y práctica de gestión pública en los hospitales. La modernización del Estado ha demandado modificar sus estructuras tradicionales y revisar su participación en la economía y su relación con la sociedad civil. Además, la importancia creciente de la participación social en las políticas públicas obliga a considerar la gestión de los hospitales desde un prisma ampliado y congruente con el desarrollo de sociedades, que subraye la transcendencia crítica, no solo de los servicios hospitalarios, sino también de los valores, sistemas, relaciones, complejidades y recursos relacionados con ellos en la sociedad. La gestión del hospital debe responder a un paradigma nuevo, complejo, e integrado de la gerencia pública, en el cual las variables de racionalidad política y los raciocinios técnicos y administrativos son elementos centrales que se completan mutuamente y conforman una nueva racionalidad gerencial (38).

Los hospitales, sean públicos o no, actúan en contextos complejos donde predominan la incertidumbre y la turbulencia, como consecuencia de factores como el acelerado cambio tecnológico, la crisis de la economía, el inusitado aumento de las demandas sociales, la permeabilidad del Estado conseguida por la sociedad y la gestión de recursos económicos. Por lo tanto, la gerencia de los hospitales, y en particular los hospitales públicos necesitan anticiparse estratégicamente a una realidad donde la complejidad, la incertidumbre y el conflicto sean retos permanentes.

Las nuevas tendencias derivadas de la nueva gerencia pública y la reforma de los sistemas de salud implican nuevos estilos de gestión, nuevas formas de financiación del sistema de salud, de tal forma que estos alcancen los objetivos y metas establecidos y satisfagan las necesidades de la comunidad. La gestión de los servicios de salud implica un amplio conocimiento de los componentes involucrados en el sistema. Estos componentes son: formas de financiación, forma de pago de los servicios, modelo de organización de servicios, política de descentralización, mecanismos de participación comunitaria, entre otros (39).

La flexibilización laboral es una de las propuestas modernizadoras de la nueva gerencia pública como estrategia destinada a contribuir a elevar los niveles de competitividad del sector público. Con ella se pretende reducir la carga presupuestaria asumida por el Estado a través de las contrataciones colectivas, 
y el crecimiento anual de la deuda interna proveniente de los pasivos que le generan al Estado su burocracia. Por otro lado, los preconizadores de este modelo alegan que la inflexibilidad laboral ha imposibilitado enfrentar el desempleo. Esto ha conllevado que una parte de las actividades públicas pueda ser realizada por profesionales que no tengan estatus de funcionarios.

En otros casos, algunas actividades son tercerizadas. Contrariamente, con base en la praxis observada, se puede afirmar que la llamada flexibilización laboral implica coartar los derechos de un sector cada vez más numeroso de trabajadores. Con ello se crean dos categorías de trabajadores o funcionarios, que aun realizando las mismas tareas, con las mismas obligaciones, un grupo goza de todos los beneficios de la contratación colectiva y la seguridad social, y otro grupo, aparte del factor inestabilidad, se encuentra por debajo de los primeros en beneficios laborales (40).

Otros señalan al factor vocacional de servicio público como primer elemento de atracción de la carrera gerencial dentro del Estado que puede ser explotado y estimulado. Asimismo, que los discursos gerencialistas se han concentrado en los incentivos económicos y la emulación de la gerencia privada. Fomentar y reconocer esa vocación constituye una garantía de probidad y compromiso (41).

En cuanto a las modalidades de contratación, es preciso destacar que las transformaciones sufridas en el aparato del Estado dieron paso a la introducción de elementos postburocráticos, lo que Ermida denomina "el paradigma de las relaciones laborales postmodernas", cuyas características, son: descentramiento del trabajo, inestabilidad en el empleo e individualización, y como resultante de lo anterior se produce el abaratamiento del costo de la mano de obra, cuya consecuencia es la reducción del salario real y se refuerza la flexibilización de los horarios en función de las necesidades productivas (42). Con esto se busca individualizar el pago y la anulación de la indemnización obligatoria por despido y el monto por antigüedad (43).

Si se utilizan suministradoras de mano de obra, ello conduce, según Ermida

a una mayor desregulación o flexibilización del Derecho del Trabajo, que amplía el ámbito de la autonomía de la voluntad del empleador y llega a tolerar que escapen de la legislación laboral hacia el Derecho Privado, 
relaciones que antes estaban indiscutiblemente reguladas por aquéllas [...] se produce cierto retroceso en las relaciones colectivas del trabajo. (44)

Con este sistema, el Estado busca "deslastrarse de compromisos laborales y asegurarse elevados niveles de productividad" (45). Además, es notable en función del agotamiento del modelo burocrático, lo que parece observarse en muchos de estos gobiernos: una mutación respecto a las formas en que tratan de cumplir sus misiones y objetivos y que los estaría acercando a modelos de nueva gerencia pública (46).

La nueva gerencia pública trae inmersa en sí la perspectiva de visión empresarial, de competencia y de rentabilidad financiera. La red hospitalaria en Colombia está recorriendo un camino en el cual su misión se ha visto inmersa en el mercado con la instauración de la Ley 100 de 1993. Ahora la rentabilidad financiera ha desplazado a la rentabilidad social, ya que con esta última no subsisten los hospitales. El actual mercado de financiación obliga a los hospitales a facturar los servicios prestados a las aseguradoras privadas y las aseguradoras públicas que tienen a su cargo los afiliados del régimen contributivo y subsidiado respectivamente. Esta cuentas médicas producto de la facturación a estas entidades son pagadas después de innumerables procesos de validación y glosas. Y todo lo anterior genera presiones en el recaudo y en el flujo efectivo de las instituciones hospitalarias (47).

Se espera que con la adopción de las técnicas de la nueva gestión pública se le estén brindando a los hospitales las herramientas para sobrevivir en el esquema de mercados. La competitividad, la productividad empresarial y la rentabilidad de estas instituciones son elementos para prevalecer en el sistema (48).

Una nueva técnica de control de gestión recientemente introducida en el sector sanitario es el cuadro de mando integral (CMI). A diferencia del presupuesto, el CMI se desarrolló para proporcionar un conjunto de información no solo financiera, sino también no financiera, que permitiera a los directivos tener una visión general de la organización sobre múltiples aspectos operativos y facilitara por ende la gestión estratégica. En los hospitales públicos, más que en cualquier otro tipo de organización, la necesidad de contemplar medidas financieras y no financieras es muy importante, ya que los hospitales tienen un conjunto adicional de valores del sector público, tales como la equidad y la justicia en el servicio. En esta línea, el CMI complementa las 
medidas financieras con medidas operativas en tres dimensiones: satisfacción del cliente, procesos internos e innovación y aprendizaje organizativo (49).

La gestión del hospital moderno necesita de indicadores de calidad que promuevan la excelencia en la prestación de servicios de salud. El modelo de excelencia de la European Foundation for Quality Management constituye un marco de referencia para la gestión de la empresa sanitaria, fomentando desde la misión y visión una cultura de innovación, tanto en los procesos organizativos como en los procesos directivos y gestores.

En el sector sanitario, el interés por esta nueva cultura de calidad ha sido estimulado por el compromiso de mejorar el valor del servicio asistencial al usuario, y además, por razones de revitalización organizativa y mejora de la eficiencia interna de la organización sanitaria, mediante resultados costoefectivos, creación de redes de aprendizaje y gestión del conocimiento operacional. La planificación y mejora de la calidad en los hospitales tanto en Europa como en el resto del mundo desarrollado se ha convertido en uno de los objetivos de la gestión sanitaria, a efectos de promover y garantizar la excelencia en los procesos de la cadena de valor asistencial. Las organizaciones hospitalarias son sistemas sociales complejos en los cuales el conocimiento como factor crítico de globalización constituye la materia prima esencial y la unidad básica de información. Estas instituciones de servicios sanitarios (hospitales) en la mayoría de los países europeos, si bien son un modelo clásico de jerarquía burocrática, no se apoyan tanto en la estructura, sino en procesos donde se materializa el conocimiento tácitamente distintivo, constituyendo en la práctica una verdadera organización operacional (50).

Se debe entender que la gestión pública, definida como la manera que el Estado obtiene, organiza, maneja y moviliza recursos escasos con la finalidad de cumplir ciertos objetivos. También ha cambiado en la última década la gestión, la cual ha venido desde una orientación burocrática enfocada en procesos, normas y procedimientos, hacia una gestión enfocada en los resultados (51).

\section{Conclusiones y reflexiones}

Las instituciones hospitalarias han atravesado y se han empoderado a través de la historia de los distintos modelo de gestión, desde el burocrático, pasando por el burocrático populista, el tecnocrático, hasta aterrizar en la nueva gestión pública de la CLAD. Todo en el contexto del cambio del papel 
de Estado, volcado hacia los sectores económicos y sociales, garantizando las condiciones favorables a la inversión privada y a la competitividad.

En el área social se destaca el papel del Estado en ser el formulador y financiador de políticas públicas y el hecho de compartir con el sector privado la ejecución de servicios públicos, principalmente los de salud y educación básica.

El nuevo modelo gerencial se basa en las transformaciones organizacionales ocurridas en el sector privado, la cuales modificaron la forma burocrático-piramidal de administración, flexibilizando la gestión y dándole más autonomía a los gerentes, pasando de una estructura basada en normas centralizadas a otra sustentada en la responsabilidad de los administradores avalados por su gestión.

Hoy la nueva gestión pública en entidades hospitalarias se basa en la administración de los recursos con fundamento y base en la racionalidad económica, que ejerce un control basado en los resultados del desempeño medidos en función de los principios de eficacia, eficiencia y efectividad, y bajo un sistema de evaluación y control de la gestión pública del ciclo gerencial PHVA, impulsados en la condición del Estado de un desarrollo acelerado y tecnología de punta para afianzar el emprendimiento y la productividad económica y social del Estado de derecho.

La mayoría de las organizaciones hospitalarias en América Latina, especialmente las públicas, buscan enmarcarse en el modelo de gestión de gerencia pública, el cual propone una gerencia basada en procesos. Esto se contrasta con lo observado en las instituciones hospitalarias en América del Norte y Europa, donde se presentan estructuras sanitarias fuertemente burocratizadas pero con gestión basada en procesos, indicadores de gestión y calidad basados en cuadros de mando integral y modelo de la excelencia EFQM de la Unión Europea, los cuales son muy parecidos a los que se presentan en la nueva gerencia pública, que permitirán a estas entidades sobrevivir en el ambiente turbulento y de incertidumbre de la gerencia actual.

Esto lleva a concluir que, independientemente del modelo de gestión, se precisará de principios de calidad aplicados a la gestión moderna que les permitan a las instituciones hospitalarias tener una visión a largo plazo de su estrategia a través de procesos vinculados al cliente y aumento del valor agregado asistencial. 


\section{Referencias}

1. Lahera E. Nuevas orientaciones para la gestión pública. Revista de la Cepal. 1994; 2:33-47. Disponible en: https://repositorio.cepal.org/handle/11362/11932

2. Diccionario enciclopédico Larousse. París: Ediciones Larousse; 1995.

3. Pérez E. La administración pública en el Estado moderno, enfoque teórico para su análisis. Ponencia en Segundo congreso argentino de administración, pública, Estado, sociedad y administración, 2003 nov 27-29; Córdoba, Argentina.

4. Martínez A. La relación entre la burocracia y las políticas públicas. Cuadernos de Dereito Actual. 2014;(2):85-98. Disponible en: https://dialnet.unirioja.es/servlet/ articulo? codigo $=4943038$

5. Weber M. Economía y sociedad. Segunda reimpresión en español. Madrid: Fondo de Cultura Económica, 2002.

6. Oszlac O. Burocracia estatal: política y políticas públicas. Posdata Revista de Reflexión y Análisis Político. 2006;11:15-56. Disponible en: https://www.redalyc. org/articulo.oa?id=52235599001.

7. Niskanen W. Bureaucracy and public economics. Aldershot: Edward Elgar Publishing; 1994.

8. Rodríguez A. Eficiencia de los hospitales públicos en España: modo de comportamiento y evidencia empírica. Revista de Economía de la Salud. 2003;804:49-50. Disponible en: https://studylib.es/doc/7989765/eficiencia-de-los-hospitales-p\%C3\%BAblicos-en-espa\%C3\%B1a

9. Molina G. Rol de los partidos políticos en la provisión de los servicios de salud, Colombia 2007-2008. Rev Fac Nac Salud Pública. 2009; 27(1):66-75. Disponible en: www.scielo.org.co/pdf/rfnsp/v27n1/v27n1a12.pdf

10. Tobar F. Hacia dónde va el hospital. Revista Medicina Global Médicos. 2018. Disponible en: https://www.revistamedicos.com.ar/opinion/hacia_donde_va_el_ hospital.pdf

11. Martin JJ. López MP. Innovaciones organizativas y de gestión en el sistema de salud. En: Temes JL, Mengibar M, editores. Gestión hospitalaria, $4 .^{\text {a }}$ ed. Madrid: McGraw-Hill; 2007. p. 25-26.

12. Ochoa H, Valladares M, Rodríguez I. Administración pública y populismo en Venezuela. RVG. 1996;1(1):39-58. Disponible en: produccioncientificaluz.org/ index.php/rvg/article/download/12722/12710

13. Pereira L, Díaz J, Pereira M, Suárez W. Influencia del modelo burocrático-populista en la gestión de la investigación: caso Universidad del Zulia. Revista de Ciencias Sociales. 2008 [citado 2018 mayo 28];14(3):614-31. Disponible en: http://www. scielo.org.ve/scielo.php?script $=$ sci_arttext\&pid $=$ S1315-95182008000300014\&l$\mathrm{ng}=\mathrm{es} \& \mathrm{nrm}=$ iso 
14. Rincón M, Rodríguez I. Descentralización y gestión de los servicios de salud en el estado de Aragua, Maracaibo. RVG. 2005;10(29):106-31. Disponible en: http://www.scielo.org.ve/scielo.php?script=sci_arttext\&pid=S1315$99842005000100006 \& \operatorname{lng}=$ es\&nrm $=$ iso

15. Molero E. Gestión de las políticas en salud en Venezuela durante el periodo 1958-2012. GT 19: Salud y seguridad social. Avance de investigación en curso. Investigación de cursante del Doctorado en Ciencias para el Desarrollo Estratégico. Mención Salud Pública de la Universidad Bolivariana de Venezuela. Disponible en: http://docplayer.es/14139890-Gestion-de-la-politica-de-salud-envenezuela-durante-el-periodo-1958-2012.html

16. Rincón M, Rodríguez I. Consideraciones generales sobre política y gestión de salud en Venezuela (1900-2003). Fermentum. 2004;14(41):503-32. Disponible en: www.saber.ula.ve/bitstream/handle/123456789/20589/articulo4.pdf? sequence $=2$

17. Estévez A. Una genealogía de la tecnocracia. En: Ochoa H, Estévez A, coordinadores. El poder de los expertos: para comprender la tecnocracia. Maracaibo: Facultad de Ciencias Económicas, Universidad del Zulia; 2006. p. 25.

18. González A. ¿América Latina, tecnocracia y centro derecha. Es posible deducir una relación inexorable entre estos factores? Trabajo preparado para su presentación en el $9^{\circ}$ Congreso de Ciencias Políticas, organizado por Asociación Latinoamericana de Ciencias Políticas, Montevideo, 2017.

19. Abadía C, Cortez G, Fino E, García C, Oviedo D, Pinilla M. Perspectivas intersituadas al capitalismo en salud. Desde Colombia y sobre Colombia. Polimpsestus. 2007/2008 May, (1):177-90. Disponible en: https://www. researchgate.net/.../228469620_Perspectivas_inter-situadas_al_capitalism

20. Hernández A, Vega R. El sistema colombiano de seguridad social en salud: desigualdad y exclusión. Revista Gerencia y Políticas de Salud. 2001 [citado 2018 mayo 28];1(1):48-73. Disponible en: http://www.redalyc.org/articulo. oa? id $=54510109$.

21. Homedes N, Ugalde A. privatización de los servicios de salud en Chile y Costa Rica [citado 2016 mayo 20]. Disponible en: www.clad.org.ve.

22. Montagut T. Política social. Una introducción. Barcelona: Ariel; 2002.

23. Hernández A, Delgado A, Márquez M, Nogueira D, Medina A, Negrín E. Generalización de la gestión por proceso como plataforma de apoyo a la mejora de organizaciones de salud. Rev Gerenc Polit. 2016;15(31): 66-87. doi: 10.11144/ Javeriana.rgyps15-31.ggpp.

24. Centro Latinoamericano de Administración para el Desarrollo (CLAD). Una nueva gestión pública para América Latina. 1998 [2008 mar. 21]. Disponible en: www.clad.org.ve. 
25. Guerrero O. Gerencia publica en la globalización. México: Porrúa; 2003.

26. Barzelay M. La nueva gerencia pública. Un ensayo bibliográfico para los estudiosos latinoamericano y (otros). Reforma y Democracia. 2001;19:7-76. Disponible en: https://cladista.clad.org/handle/123456789/1162

27. Ochoa H. Gobernaciones y nueva gestión pública en Venezuela. Revista de Servicio Público. 2003;54(1):7-97. doi: 10.21874/rsp.v54i1.263.

28. Ormond D, Löffler E. Nueva gerencia pública: ¿qué tomar y qué dejar? CLAD Reforma y Democracia. 1999;13:1-20. Disponible en: https:/cladista.clad.org/ handle/123456789/476

29. Blutman G, Cao H. Alimentando el debate en la administración pública en estado y administración pública. Buenos Aires: Centro de Investigaciones de Administración Pública, Universidad de Buenos Aires; 2016.

30. Longo F, Echevarría K. La nueva gestión pública en la reforma del núcleo estratégico del Gobierno: experiencias latinoamericanas. En: Spink P. Nueva gestión pública y regulación en América Latina. Balances y desafíos. Caracas: Centro Latinoamericano de Administración del Desarrollo, Editorial Texto. p. 97-112.

31. Kotler RE. Marketing social: estrategias para cambiar la conducta pública. Madrid: Díaz de Santos; 1991.

32. Ochoa H. Consideraciones en torno al fenómeno de la tecnocracia. Cuestiones Políticas. 1993;11:17-39.

33. Naranjo D. El uso del cuadro de mando integral y del presupuesto en la gestión estratégica de los hospitales públicos. Gac Sanit. 2010;24(3):220-4. Disponible en: https://dialnet.unirioja.es/servlet/articulo?codigo $=3422598$

34. Kliksberg B. Una nueva gerencia pública para la modernización del estado y afrontar los desafíos de la integración [citado 2008 ago. 24]. Disponible en: http:// ciber-economia.iespana.es

35. Córdova E. Modernización de la infraestructura de servicio a la economía por parte de las gobernaciones en Venezuela: el caso del estado Zulia. Gerencia Pública. 1999;4(7):71-98. Disponible en: https://cladista.clad.org/handle/123456789/829

36. Suárez C, Márquez Y, Nava Y, Ojeda A. Relaciones de trabajo en el contexto de la globalización. RVG. 2001;6(15):382-401. Disponible en: http:// produccioncientificaluz.org/index.php/rvg/article/view/9080

37. Sarabia E. La situación del personal de la administración. Seminario sobre la administración pública en América latina, 24 y 25 de abril de 1997, CLADPNUD, 1998. 
38. Bresser L. De la administración pública burocrática la gerencial. Caracas. CLAD, Estado, Administración Pública y Sociedad. 1996; 2:16. Disponible en: unpan1. un.org/intradoc/groups/public/documents/CLAD/CLAD15813.00.pdf

39. Organización panamericana de la salud. El escenario global de la nueva gestión del hospital en la transformación de la gestión de los hospitales de América Latina y del Caribe. Washington: Biblioteca Sede OPS; 2001. p. 14-33.

40. Molina G. Tendencia en la gestión de los servicios de salud. Revista de la Facultad Nacional de Salud Pública. 2001;19(1):71-89. Disponible en: https://www.redalyc. org/articulo.oa?id=12019106

41. Córdoba E. Administración pública en Venezuela: aproximaciones a los cambios y transformaciones. Revista de Ciencias Sociales. 2006;12(3):496-518. Disponible en: https://www.redalyc.org/articulo.oa?id=28014478007

42. Tohá C, Solari R. La modernización del Estado y la gerencia pública. Nuevas tendencias en la modernización del Estado. 1996. Disponible en: www.fes.org.ar

43. Laurell AC. Structural adjustment and the globalization of social policy in the Latin America. International Sociology. 2000;15(2):306-25. Disponible en: https:// doi.org/10.1177/0268580900015002010

44. Ermida O. Globalización y relaciones laborales. Revista Venezolana de Gerencia. 1999;4(9):135-48. Disponible en: http://produccioncientificaluz.org/index.php/ rvg/article/view/8950

45. Minayo C, Da Fonseca S. Descentralización y participación en salud: nuevos partes para la discusión. En: Briceño R, De Souza Minayo M, Coímbra C, coordinadores. Salud y equidad: una mirada desde las ciencias sociales. Caracas: Editora Fiocruz; 2000. p. 15-24

46. Torres J. Innovación en los gobiernos locales en Iberoamérica. Posibilidades a partir de la nueva gerencia pública. Estudios Gerenciales. 2012 [2018 mayo 30];28:281-302. Disponible en: http://www.redalyc.org/articulo. oa?id $=21225840015$

47. Ochoa H, López M, Rodríguez I. Administración pública y populismo en Venezuela. Revista Venezolana de Gerencia.1996;1(1):39-58. Disponible en: www. produccioncientifica.luz.edu.ve/index.php/rvg/article/view/12722

48. Avendaño J. Análisis de la nueva gerencia hospitalaria. Un enfoque de gestión administrativa dentro del sector hospitalario [trabajo de grado maestría en Ciencias Económicas]. Bogotá: Universidad Nacional de Colombia; 2010.

49. Narango G. el uso de cuadro de mando integral en el presupuesto y en la gestión estratégica de los hospitales público. Gac Sanit. 2010;24(3):220 4. Disponible en: http://scielo.isciii.es/scielo.php?script=sci_arttext\&pi$\mathrm{d}=$ S0213-91112010000300008\&lng $=$ es 
50. Oteo L, Pérez G, Silva D. Cuadro de mandos integral a nivel hospitalario basado en indicadores del modelo EFQM de excelencia. Revista Gestión Hospitalaria. 2002;13(1):9-25. Disponible en: campus.easp.es/recursos/DiplomaGestion/documentos/cuadromandos.pdf

51. Waissbluth M, Larraín F. Modelos de gestión pública: implicancias para la planificación, evaluación y control de gestión del Estado. En: Un mejor Estado para Chile. Propuestas de modernización y reforma. Santiago de Chile: Consorcio para la Reforma del Estado; 2009. p. 541-59

\section{Notas}

1 El mercado significa otorgar a los consumidores de los servicios públicos una mayor influencia, mediante la ampliación de los derechos de elección, información, participación y reclamación (30, p. 104). 